Claude Muller

Université Bordeaux-Montaigne et CLLE France

(iD https://orcid.org/0000-0002-7355-1765
Négation, syntaxe, détermination. Un bilan et des questions

\title{
Negation, Syntax, Determiners.
} An evaluation and some questions

\begin{abstract}
The present paper retraces some results of my own investigations, among other authors, on such questions as negation, levels of analysis of sentences, and internal structure of NPs, after years of research in the field. It suggests some possible paths of further investigations, among others: thus, negation needs a multi-levels of analysis, including an enunciative component; syntax requires an adequate description of informative value of items, and the internal composition of determiners in French remains an open question.
\end{abstract}

\section{Keywords}

Negation, syntax, predicative structures, determiners, indefinites, contrastive analysis

\section{Introduction}

Comment aborder, pour un linguiste à peu près au terme de sa carrière, la question que pose Gaston Gross, d'une vision prospective du domaine, ou plus modestement, de ce que pourrait être la recherche à mener dans les parties précises du champ des recherches linguistiques qui sont celles que nous avons pu, chacun à sa manière, parcourir pendant quelques décennies ? Vaste question, qui demanderait beaucoup plus de place qu'un bref article pour tenter d'y répondre. Il faudra sans doute du temps pour que l'immense masse de publications de la linguistique de la fin du $\mathrm{XX}^{\mathrm{e}}$ siècle, après décantation, laisse apparaître ses avan- 
cées au-delà des débats théoriques qui ont souvent occupé le devant de la scène, mais celles-ci sont certainement considérables : que l'on compare par exemple, ce qu'était la syntaxe générale du temps de Martinet (1985) à ce qu'elle est devenue chez Creissels (2006), ou, dans un autre domaine, l'état de la sémantique du français avant et après Georges Kleiber, pour me limiter à la production scientifique en français. Pour tenter d'y répondre, je vais m'appuyer sur un état des lieux très parcellaire, celui de quelques-unes des questions que j'ai abordées depuis les presque cinquante années que je publie des travaux en linguistique ${ }^{1}$. On ne trouvera donc pas ici l'argumentation classique d'une investigation sur un domaine précis, mais une sorte de bilan, au-delà duquel j'espère tracer en pointillés des pistes de ce que serait ma recherche à venir si j’avais la longévité de Mathusalem, ou ce que je conseillerais à un jeune linguiste de faire s'il épousait ma manière de voir et de faire en linguistique. Vœux pieux, dira-t-on, puisque les temps ont changé, et aussi les conditions dans lesquelles s'élaborent actuellement les recherches dans notre domaine. Je ne peux que regretter l'époque, vers le début des années 70 de l'autre siècle, où la linguistique avait pour elle l'attrait de la nouveauté, attrait justifié par une forme de sclérose de la recherche grammaticale française, et puissamment développé par les courants innovants venus d'outre-Atlantique, l'enthousiasme de jeunes chercheurs, conjugués à Paris avec la renaissance universitaire qui suivait mai 68 et la fondation de l'Université de Vincennes, et où il y avait des enseignants jeunes, brillants et atypiques, Richard Kayne, Maurice Gross et Nicolas Ruwet, et un public fervent pour accueillir ces travaux et les faire avancer. Les temps ont bien changé, où il s'agissait d'une discipline émergente, qui devait lutter pour une place au soleil, ce dont témoigne pour les nostalgiques de cette époque et de cette atmosphère le livre de Jean-Claude Chevalier, Combats pour la linguistique, dont le titre même paraît bien anachronique en 2021. J'examinerai ici trois domaines, qui sont d'ailleurs liés comme on verra, sur lesquels se sont portées mes recherches.

\section{La négation et la modularité des grammaires}

Mes premières recherches, pour un doctorat de $3^{\mathrm{e}}$ cycle soutenu en 1975, intitulé Négation et quantificateurs, portaient sur les interactions de portée de ces opérateurs, négation d'une part, déterminants et pronoms indéfinis de quantité d'autre part, avec pour conséquence, pour ces derniers, des modifications importantes dans le sens, par exemple quelqu'un interprétable selon les contextes, comme renvoyant à un individu identifiable, ou bien à une pluralité indéterminée :

\footnotetext{
${ }^{1}$ Voir mon site Claude Muller-linguiste.
} 
Je viens de m'apercevoir que quelqu'un nous écoute Je ne pense pas que quelqu'un puisse nous entendre

On y reconnaît la question des relations de portée ou de scope, et celle du changement dans l'extension des indéfinis selon leurs interactions avec des négations. Il existait dans la linguistique française une théorie d'accueil pour examiner ces phénomènes, celle de Damourette et Pichon (1911 - 1940), avec en guise de portée de la négation l'« atmosphère forclusive », et son explication, permettant d'ailleurs des analyses de qualité, comme celle de Robert Martin (1966) sur le mot « rien ». Mais l'interprétation psychologique de Damourette et Pichon, en particulier leur analyse de la combinaison des deux marques de la négation du français, ne et pas, en termes sémantique de «discordance » pour la première et de " forclusion » pour la seconde, semblait peu convaincante. Autrement solide était la tradition structurale, illustrée brillamment pour la négation par David Gaatone (1971), mais elle restait purement descriptive. C'est la linguistique américaine, à partir de Klima (1964), qui a fait le lien entre la description syntaxique des domaines de portée et la variation morphologique des adverbes, pronoms et déterminants non définis selon les contextes, formant des séries plus visiblement apparentées en anglais qu'en français (somebody, anybody, nobody), et l'interprétation sémantique distinguée par les « indéterminés » (les séries some) et les " indéfinis » (les séries any), les " négatifs » incorporant la négation. Dans ces analyses, la négation impliquait une théorie syntaxique des domaines de portée, une analyse des incorporations sémiques à l'interface de la morphologie et de la syntaxe, et une analyse sémantique permettant non seulement de constater, mais d'expliquer, pourquoi les indéfinis changeaient de sens, ou de forme, selon les contextes. Les théories chomskyennes de l'époque fournissaient un cadre explicatif et permettaient de décrire des domaines syntaxiques dans les frontières desquelles un opérateur comme la négation pouvait exercer son influence sur l'interprétation et l'occurrence lexicale des indéfinis. À l'époque, l'opposition cristallisée sur la « sémantique générative » (G. Lakoff, J. McCawley²), d'une part, et la « théorie standard» de l'autre (R. S. Jackendoff, 1972) d'autre part, masquait la convergence profonde des deux approches : la négation et les autres phénomènes régissant l'interprétation, la forme et la référence des indéfinis, nécessitaient une analyse en niveaux distincts de l'énoncé, celle de structures de type prédicat/ argument d'une part, et d'autre part celle de la syntaxe " de surface », avec des possibilités de renversement de l'interprétation attendue. Un exemple :

Quand elle n'appréciait pas quelqu'un, nulle comédie : elle tirait à vue et s'éloignait à jamais. (Fr. Nourissier, À défaut de génie, 2000, p. 69)

\footnotetext{
${ }^{2}$ Voir les articles dans Steinberg et Jakobovits, 1971.
} 
Les règles de choix et d'interprétation des indéfinis dans la dépendance syntaxique directe de la négation devraient imposer ici la forme personne, pourtant exclue. Mais la phrase doit se comprendre comme une construction existentielle soumise à un opérateur imposant une interprétation « habituelle », ou répétitive, contenant l'indéfini, qui reste cependant hors de la portée de la négation :

Quand il y avait quelqu'un (qui que ce soit) qu'elle n'appréciait pas, nulle comédie...

Cette glose reflète l'organisation prédicative de l'énoncé, et rend compréhensible la forme et le sens de quelqu'un dans cette phrase : l'indéfini est bien hors de portée de la négation, porte une valeur prédicative existentielle, et dépend pour son interprétation de la démultiplication des contextes d'occurrence possibles créée par quand; de ce fait, quelqu'un n'est pas spécifié par son contexte autant qu'il le serait dans une phrase épisodique ancrée dans le réel, et la série qui que ce soit n'est pas exclue, ou pourrait au moins figurer en apposition. La compréhension des relations de portée exige donc un niveau d'analyse de l'énoncé où ce qui apparaît comme un syntagme nominal indéfini sujet ou complément d'un verbe est en réalité le représentant d'une proposition.

Les exigences de description de la phrase négative m'ont conduit par conséquent à adopter une analyse de l'énoncé qui prenne en compte ce fonctionnement prédicatif, qu'il soit représenté par une arborescence syntaxique comme le proposait la " sémantique générative ", comme une « forme logique » dans les autres théories chomskyennes, ou une organisation prédicative sous-jacente, dans les grammaires à opérateurs de Z. Harris ${ }^{3}$. J'y reviendrai un peu plus loin.

D'une façon plus générale, l'analyse de la négation oblige à une vision globale de la description linguistique des énoncés, pas seulement à la description grammaticale de la phrase. Elle met donc en question les analyses purement syntaxiques, comme l'étaient dans leurs débuts les descriptions chomskyennes. J'ai décrit ceci dans un article qui met en évidence cette exigence de profondeur, ou de démultiplication des niveaux d'analyse, dans « La négation, un opérateur transversal » (Cl. Muller, 2008). On ne peut traiter de cette question sans examiner les questions de sens et de praxis, ce qui fait de la négation un marqueur énonciatif et pas simplement un quelconque adverbe de phrase. La négation brouille les frontières des sous-catégories descriptives habituelles de la grammaire : en termes morpho-lexicaux, les «négatifs » relèvent à la fois des " parties du discours " traditionnelles, et de cette superstructure qui a ses propriétés, comme les marques de l'interrogation ou celles de l'exclamation. Prendre comme objet d'étude la négation, c'est donc s'obliger à inclure dans la description une théorie

${ }^{3}$ La théorie transformationnelle de Harris est exposée dans de nombreux textes, voir Harris, 1991 ; en français, Harris, 1976. 
de l'énonciation, et une représentation du sens incluant les notions de présupposés, de points de vue, une pragmatique des énoncés. On peut s'en rendre compte par exemple dans l'examen des différences entre les interprétations " polémiques » ou « métalinguistiques » de la négation par rapport à la négation « descriptive » (O. Ducrot, 1984 ; H. Nølke, 1992). Non seulement la négation oblige à inclure dans la grammaire une théorie des présuppositions, mais il faut aussi que ces présuppositions soient adossées à des points de vue énonciatifs différenciant le point de vue du locuteur, celui d'interlocuteurs réels, ou encore celui que le locuteur attribue aux croyances communes. En cela, l'étude de la négation oblige à aller audelà du sens, à s'intéresser à l'aspect « comportementaliste » du langage, comme le déclare Pierre Attal (1994).

Dès lors, se pose la question du cadre théorique adopté. À l'époque de mes premiers travaux sur la négation et les quantificateurs, qui se sont poursuivis par la suite en une thèse sur la négation en français ${ }^{4}$, Pierre Attal travaillait sur le même sujet, alors que nous étions tous deux enseignants à Rennes, et a soutenu une thèse d'État sur la négation et les quantificateurs (P. Attal, 1979) à Paris-8, dans l'optique des travaux sur les actes de langage et l'argumentation, alors que je travaillais d'abord dans une optique générative, puis sous la direction de Maurice Gross. Rien ne m'a mieux fait comprendre comment la diversité des approches, ou des points de vue, cette fois du linguiste, pouvait orienter la recherche en linguistique au point de rendre les analyses, parfois, incompatibles. Pourtant, nous avions en commun un intérêt initial pour les travaux de Chomsky ${ }^{5}$, et nous avons tous deux assez rapidement abandonné les évolutions de la théorie chomskyenne, ses zigzags et ses excommunications. De plus, la négation ne pouvait entrer dans une théorie centrée sur la seule syntaxe, avec une sémantique d'interprétation. Le cadre théorique beaucoup moins élaboré de Zellig Harris, en outre proposé comme source théorique de son approche par mon directeur de recherches, Maurice Gross, m'a toujours semblé, un peu comme une esquisse d'une théorie de type sens-texte avant l'heure, mieux à même de proposer un cadre plus approprié à l'analyse des langues, avec sa structure de l'information en relations à deux catégories basiques, le prédicat et l'argument. C'est sur ce canevas que j'ai construit mon cadre de description syntaxique dans Les bases de la syntaxe (2002-2008). Cependant, Pierre Attal découvrait pendant la même période les travaux de Ducrot : ses analyses s'orientèrent rapidement vers les effets argumentatifs de la négation, l'étude des lois d'« abaissement » de la quantification, ainsi que vers le postulat d'un acte de "refus » faisant de la négation polémique de Ducrot, le type central de la description illocutoire. Attal rejoignait ainsi la position de

${ }^{4}$ Ma thèse, soutenue en 1987 à l'Université de Paris-7, est en partie reprise dans mon livre de 1991 : La négation en français, Droz, Genève.

${ }^{5}$ Attal a traduit en français l'ouvrage générativiste de Kayne (1975), French Syntax devenu Syntaxe du français. Il a assuré à Rennes un cours intitulé « Grammaire transformationnelle », dont j’ai pris la suite dans les années 1975 à 1987. 
Searle (1972) proposant un acte illocutoire de négation. Sans méconnaître ces aspects, mes travaux me conduisirent plutôt vers les interactions entre la négation et les autres constituants prédicatifs de l'énoncé, notamment ceux modifiés sémantiquement et parfois lexicalement par la portée de la négation, les adverbes et les indéfinis « négatifs » et leur interaction syntaxique avec la négation verbale. Pour en revenir à la grammaire, il m'a semblé que si la négation pouvait être un marqueur énonciatif ayant effectivement la valeur d'un refus ou d'un rejet, elle était dans d'autres situations un élément combinatoire articulé avec d'autres marqueurs énonciatifs, comme l'assertion : il y a évidemment assertion de la négation, prise en charge par le locuteur dans les énoncés polyphoniques, enfin la négation combinée à l'interrogation, par exemple dans la locution de demande d'adhésion n'est-ce pas? du français, n'est absolument pas analysable comme un refus.

Le rejet pur et simple, sans réinvestissement dans l'assertion de la négation et dans un énoncé négatif qui fasse sens, est peut-être assez rare. Sa manifestation typique serait dans le Non! qui échappe au locuteur lors de l'annonce d'une mauvaise nouvelle ou de la découverte d'une situation intolérable. Dans les autres cas, que la négation soit polémique ou métalinguistique, le rejet construit du sens, un sens lacunaire certes, mais asserté comme tel par le locuteur. Il y a une information véhiculée, par exemple dans :

\section{Paul n'a pas été reçu à son examen}

ou encore un ordre donné, dans :

\section{Tu n'iras pas danser ce soir}

et en définitive, la négation s'intègre aisément dans le même moule de phrase que l'assertion, phrase que l'on appelle quelquefois « déclarative ».

En définitive, j'ai adopté le point de vue inspiré par Frege de la négation comme " jugement ", en-deçà de l'acte de langage austinien, mais combinable avec lui. La négation se différencie des prédicats usuels en ce que, à strictement parler, elle n'a pas de sens, comme le disait Wittgenstein (1961) à propos de l'opérateur logique de négation : «Au signe $\neg$ rien ne répond dans la réalité ». Un « jugement » est dans l'optique frégéenne une appréciation de l'énonciateur sur l'adéquation ou non de tel ou tel prédicat au regard du réel, du souhaité, du normal, ou du dit antérieur, jugement à distinguer de la partie performative qui caractérise un acte de langage. Dans l'énoncé affirmatif non marqué, rien n'indique qu'une telle opération ait lieu : l'énoncé est construit par l'assemblage des différents prédicats, coulés dans la syntaxe de l'assertion, qui est la construction non marquée de l'énoncé. L'analogue de la négation dans le domaine de l'affirmation serait le jugement de confirmation d'un point de vue extérieur ou déjà exprimé : 
ce qu'indique en français l'adverbe bien, et que l'on peut caractériser comme un acte de « confirmation» :

\section{Il a bien accepté de répondre à nos questions}

Il y a donc une asymétrie entre l'énoncé négatif et l'énoncé affirmatif dans sa forme usuelle ${ }^{6}$. De plus, le jugement négatif a sa spécificité. On sait depuis longtemps que la négation, liée à une perception moins aisée du réel (T. Givón, 1978), à une attente contrariée, à une situation jugée inadéquate, véhicule de la négativité, au sens psychologique. Elle construit aussi des contextes d'interprétation particuliers, où les implications sont renversées, où les indéfinis, souvent marqués morphologiquement, sont plus indéterminés dans leur référence, ce que l'on a appelé la polarité négative. L'énoncé négatif n'est donc pas non plus une simple variante de l'assertion.

Quel est actuellement le bilan des études sur la négation, et quels domaines devraient être plus particulièrement examinés ? On peut, on doit, évidemment, utiliser le travail monumental de Laurence R. Horn, A Natural History of Negation, si possible dans la version augmentée de 2001 plutôt que l'originale de 1989, pour avoir une idée des innombrables travaux consacrés non seulement en linguistique, mais aussi en philosophie du langage et en logique, sur cette notion. On peut aussi lire avec profit l'étude critique qu'a faite Pierre Attal (1992) de ce livre. Dans le domaine purement linguistique, il faudrait aussi, comme toujours maintenant, consulter les études typologiques sur la négation, comme Kahrel et van den Berg (1994), à compléter par des recherches plus récentes. En ce qui me concerne, je suis revenu sur la négation, dans ses relations avec la référence (Cl. Muller, 2017) : on a toujours tenté, depuis Platon avec le concept d'altérité (dans le Sophiste) de donner un contenu positif au prédicat nié. On connaît la tentative de Katz (1972) consistant à interpréter positivement un énoncé négatif comme Ce n'est pas une pizzeria en utilisant la notion de complémentaire dans le même domaine, mais ce recours à la pragmatique suppose un usage " raisonnable » du langage, non la réalité extrêmement diverse des interprétations que des phrases de ce type peuvent recevoir dans leur contexte. Que l'on songe à la phrase titre du tableau de Magritte, Ceci n'est pas une pipe, on se rend compte que l'interprétation par le complémentaire est tout à fait réductrice, et tient non à la négation, mais à un type courant mais pas généralisable ni inscrit dans la sémantique de la phrase d'interprétation pragmatique. Il en va de même avec les interprétations basées sur la notion de présupposé, associé à une analyse syntaxique.

${ }^{6}$ Certaines langues ont un marqueur d'assertion affirmative : c'est le cas du gascon, ou du basque. Cependant, ce marquage, limité aux principales ou indépendantes, soit n'apparaît que dans certains contextes (basque, voir ci-dessous), soit n'est pas généralisé dans tous les sous-dialectes. En gascon, son usage généralisé se limite à la zone pyrénéenne, et reste sporadique ailleurs, ce qui signale qu'il n'est pas absolument indispensable. Sur cette question, voir Muller, 2008. 
Dans son étude sur la portée de la négation (1981), Cristina Heldner s'intéressant à des énoncés comportant une proposition finale montre que la négation cible souvent celle-ci, laissant hors de sa portée le verbe principal :

Ils n'étaient pas venus là pour discuter (op. cit., p. 142)

$=$ Ce n'est pas pour discuter qu'ils étaient venus (donc : ils étaient venus)

mais aucune règle n'impose cette interprétation, comme le montre un autre de ses exemples :

L'auteur n'était pas là pour défendre son cuvre mais on l'excusait : il venait d'être assassiné. (ibid.)

Dans ce cas, il est impossible, du fait de la coordonnée qui suit, d'avoir le même schéma de portée que dans la phrase précédente : il faut comprendre que la négation porte sur la principale, et que la subordonnée finale est incluse dans la présupposition : " on s'attendait à ce que l'auteur soit là pour défendre son œuvre », et c'est donc cet ensemble qui est l'objet de la négation. Autrement dit, on ne peut déduire de la syntaxe ou de la compositionnalité ou de la pragmatique des domaines lexicaux des règles stables et générales, hors contexte, d'interprétation « positive » des énoncés négatifs.

\section{L'analyse grammaticale en grammaire contrastive}

Mes centres d'intérêt m'ont donc conduit à développer des analyses sur la structure de la phrase, analyses basées essentiellement pour moi sur le français dans un premier temps, travaux sur les clivées, la subordination en français (1996), les clitiques (Cl. Muller et al., 2000), sur l'ordre des mots (K. Gerdes, Cl. Muller, 2006). J'ai rassemblé en un volume de synthèse ces travaux, complétés d'analyses contrastives, avec l'aide de spécialistes de chaque langue étudiée, mettant en évidence des structures syntaxiques différentes dans les langues voisines géographiquement du français, allemand, anglais, langues romanes, parmi lesquelles une langue régionale, l'occitan gascon, breton, et basque. Il en est résulté Les bases de la syntaxe, ouvrage dans lequel les structures propres au français sont comparées dans un même modèle d'analyse à ces autres langues.

Dans ce modèle syntaxique d'analyse que j'ai développé, il me semble possible de donner une description assez précise des langues du monde, parce que le cadre est assez souple et général pour que les langues les plus diverses y soient intégrables. Comme je l'ai dit ci-dessus, la base théorique doit beaucoup à Maurice 
Gross et à l'école du Lexique-Grammaire qu'il a fondée, ainsi qu'à Zellig Harris et à son modèle transformationnel mettant l'information à la base de sa description de la grammaire, mais elle repose aussi sur un corpus commun d'analyses de la fin du siècle précédent, analyses que je considère comme des acquis de la théorie chomskyenne, indépendamment des variations de celles-ci. De plus, comme je l'ai dit plus haut, mon domaine de recherches, la négation, nécessitait de placer dans la grammaire des énoncés, au point de départ du vouloir-dire du locuteur, une composante énonciative, et de prendre en compte dans la bonne formation des énoncés des aspects pragmatiques tels que les présupposés.

Cet échantillon de langues est suffisamment riche de données divergentes pour comprendre que la syntaxe est, dans une grammaire modulaire, le niveau le plus diversifié qui soit, alors que l'analyse des structures prédicatives est assez uniforme, du moins tant que l'on ne sort pas des langues européennes. Au niveau syntaxique, c'est-à-dire dans la structuration de la phrase en syntagmes hiérarchisés, le français, langue $\mathrm{SVO}^{7}$ sans cas morphologiques est ainsi confronté à une langue VSO, le breton, avec une position de topicalisation/focalisation initiale dans les indépendantes, mais à verbe initial dans les subordonnées; une langue de type Topique/focus-VSO, avec un système casuel, l'allemand, avec une structure asymétrique, les subordonnées étant de type SOV ; et le basque langue ergative $^{8}$, non configurationnelle, c'est-à-dire sans fonction attribuable par l'ordre des constituants nominaux actants, et probablement sans syntagme verbal.

La syntaxe met en évidence un ordre des mots dicté par le marquage fonctionnel, notamment dans les langues sans cas, et souvent, un autre type de structure $\mathrm{y}$ figure et s'y superpose, le marquage des fonctions communicatives, notamment la focalisation et la topicalisation. Les langues non configurationnelles, comme le latin ou le basque, ont un ordre dit neutre, qui est simplement la réalisation la plus courante des contraintes communicatives. Ainsi, dans le cas du basque, malgré la liberté de position des actants, la structure usuelle est décrite comme SOV, description discutée, puisque les positions $\mathrm{S}$ et $\mathrm{O}$ ainsi indiquées sont respectivement des positions de topique et de focus, et que l'une des rares contraintes de position du basque exige que la position qui précède le verbe conjugué soit un terme focalisé. L'analyse SOV tient à ce que beaucoup de phrases du basque présentent un sujet initial topique, suivi d'un objet qui est normalement focalisé, mais il est tout à fait possible de réaliser un énoncé à verbe initial, si le verbe est comme c'est le cas le plus souvent dans sa construction analytique, avec la distinction de la forme lexicale du verbe, et de l'auxiliaire portant les marques d'actance et de temps. Ainsi, on trouvera aussi bien la construction usuelle, SOV

\footnotetext{
${ }^{7}$ Pour sujet, verbe, objet.

${ }^{8}$ En basque, le sujet intransitif est à l'absolutif, cas non marqué, comme l'est le complément d'objet direct, alors que le sujet des verbes transitifs est marqué (cas ergatif).
} 
Peio Bilbotik etortzen da9.

Pierre Bilbao+de venant 3sg-est

«Pierre vient de Bilbao »

que la construction sans actant nominal, avec le verbe lexical dans la position de focus obligatoire :

\section{Etortzen da.}

venant 3sg-est

«Il/Elle vient »

Dans la construction synthétique du verbe, cette phrase serait impossible, du fait de l'absence d'auxiliaire pour occuper la position de focus ; le même verbe « venir » dans la conjugaison synthétique donne l'énoncé inacceptable suivant :

\section{*Dator}

3sg-vient « il/elle vient »

On y remédie à l'affirmatif en plaçant à l'initiale une particule énonciative $b a$ avec ce rôle de support de focus :

\section{Badator gero aitxitxa.}

EN-3sg-vient plus-tard grand-père,

" Grand-père vient plus tard » (J. I. Hualde et al., 2003, p. 81).

Ce préfixe est relié au mot servant à l'affirmation, bai (« oui »). Dans la conjugaison analytique, il occupe la position de focus avec interversion du verbe lexical et de l'auxiliaire, permettant une affirmation emphatique (ibid., p. 538) :
Erosi $d u$
Badu erosi
acheté 3sg-l-a
EN+3sg-l'a acheté
« Il/Elle l'a acheté »
« I1/Elle l'a acheté ! »

Avec la conjugaison synthétique, la négation occupe la position de la particule énonciative et rend la phrase acceptable :

Ez dator

NEG 3sg-vient « Il/Elle ne vient pas »

\footnotetext{
${ }^{9}$ D’après Rebuschi, 1990. Aussi Hualde et Ortiz de Urbina, 2003.
} 
On remarque dans ce cas que la négation occupe la place d'une particule d'affirmation, ce qui confirme ce que l'on a dit plus haut sur le fonctionnement énonciatif de la négation. Un autre trait de syntaxe va dans le même sens : dans la conjugaison analytique, la négation modifie l'ordre structurel sans marquer d'emphase, se plaçant devant le verbe conjugué antéposé, comme pour l'emploi emphatique de $b a$ :

\section{Ez du erosi \\ NEG 3sg-l-a acheté \\ « II/Elle ne l'a pas acheté »}

Ce qui distingue la basque d'autres langues, c'est que l'on reste toujours, une fois respectée la contrainte minimale d'une position de focus devant le verbe conjugué, en droit de placer les actants, en ordre libre, sans aucun marquage communicatif sur eux, hors du domaine de focalisation, comme le montre Rebuschi (1990) :

\section{Etortzen da Peio Bilbotik / Etortzen da Bilbotik Peio. \\ Venant 3sg-est Pierre Bilbao+de \\ «Pierre vient de Bilbao»}

On voit par l'exemple du basque l'importance de l'intrication des facteurs communicatifs dans la syntaxe, puisqu'en définitive, le basque montre que la position de focus doit être attribuée en toutes circonstances, et que l'on y supplée à défaut par une particule énonciative liée à l'assertion. D'autre part, la liberté de position des actants basiques de l'énoncé exclut, à mon sens, d'y trouver une justification à la représentation d'un "syntagme verbal » en tant que structure syntaxique associant verbe et compléments, hypothèse qui, plus que dans d'autres langues, paraît bien peu justifié ici. Une des pistes prometteuses de recherche consisterait sans doute, sur un vaste échantillon de langues de divers types, d'examiner les modes d'intégration des facteurs communicatifs dans la syntaxe.

Les langues romanes, plus proches du français, offrent aussi un domaine d'étude privilégié, celui des clitiques pronominaux, avec des règles de positionnement complexes, plus ou moins rigides, que l'on a tenté de décrire selon des règles d'optimalité, avec une évolution en diachronie qui va vers un ordre figé pour le français, alors que les langues ouest-ibériques, portugais, galicien, asturien, comportent encore une grande diversité de placement, conditionnée par de multiples facteurs.

Dans mes cours, j'ajoutais à l'étude des langues mentionnées ci-dessus notamment le japonais et le chinois mandarin, pour étudier d'autres structures, par exemple la différenciation du sujet et du thème, marquée en japonais, ou encore une langue sans sujet obligatoire, comme le chinois. On trouve aussi, en chinois, 
des structures prédicatives différentes de celles auxquelles on est habitué, du fait de constructions de type sériel peu répandues dans nos langues (D. Creissels, 2006 , t. 2, §37-4 à 37-6). Ces constructions peuvent aussi mettre en question la séparation que l'on fait entre subordination et juxtaposition ou coordination sans marque, les constructions sérielles étant utilisées pour décrire des événements liés, sans précision sur le type de lien. Par exemple, le chinois a de nombreuses constructions dans lesquelles la juxtaposition des verbes ne permet pas directement de savoir s'il y a coordination de deux événements concomitants ou subordination implicite, généralement vue comme finale ou habituelle (C. N. Li, S. A. Thomson, 1989, chap. 21).

Ainsi, en chinois, la construction suivante ${ }^{10}$ peut s'interpréter de deux façons :

A Jiu chuan tuoxie shang-ke

NPr porter tong aller-cours

« A Jiu porte des tongs et va en cours avec »/ « A Jiu met des tongs pour aller en cours »

Dans la première traduction, on voit que l'interprétation prédicative est celle d'une coordination avec une relation temporelle commune, non marquée ; dans la seconde, le second verbe correspond à une subordonnée. Il y a donc dans le second cas une dépendance prédicative, comme dans les équivalents français, dans lesquels la subordonnée est prédicat dominant avec le verbe principal comme argument, mais dépendant syntaxiquement, alors que dans l'interprétation coordonnée les deux verbes sont indépendants l'un de l'autre.

Il y a cependant une contrainte qui lève cette apparente indifférenciation entre subordination et coordination dans cette construction : il s'agit de la possibilité d'utiliser un des rares marqueurs temporels/aspectuels du verbe en chinois, la particule le indiquant l'accompli. Dans l'interprétation coordonnée, pour indiquer une action épisodique passée, la particule le signifiant « accompli » est de mise sur le verbe principal, mais peut aussi, facultativement, s'adjoindre au verbe coordonné. Sa présence dans ce cas est une marque d'insistance sur l'interprétation épisodique passée :

\section{A Jiu chuan-le tuoxie shang-(le)-ke}

NPr porter-acc. tong aller-(acc.)-cours

«A Jiu s'est mis des tongs et il est allé en cours avec»

Dans la construction à sens de subordination, on ne peut pas utiliser le marqueur le sur le second verbe : il y a donc bien, malgré les similitudes, une différence dans ce cas particulier entre subordination et coordination.

${ }^{10}$ Exemple tiré de Mallet-Jiang (2012). On omet le marquage tonal, non pertinent. 
Il reste évidemment beaucoup à faire dans la description contrastive des grammaires. Si je devais poursuivre ce travail, je continuerais ces examens contrastifs dans la même perspective ${ }^{11}$, centrée non comme trop souvent sur le désir de pousser en avant une nouveauté théorique, ou la mise en évidence d'une hypothétique grammaire universelle, mais sur les traits et domaines qui caractérisent la grammaire d'une langue, et par induction, sur les modules et règles que la description doit accueillir.

\section{La détermination nominale, les indéfinis et les partitifs}

Je suis revenu récemment (Cl. Muller, 2019) à un objet d'études qui m'a toujours intéressé, la construction des déterminants du français, et particulièrement les constructions indéfinies, parmi lesquelles figurent les quantifieurs, mais aussi les constructions partitives. Contrairement aux déterminants définis, à base du le article, les indéfinis offrent, en plus de leur caractérisation sémantique, qui est précisément l'indéfinition de la référence du syntagme qu'ils introduisent, une grande diversité de formes et de structures. Je vais donc essayer de préciser cidessous les résultats auxquels je pense être parvenu, et les interrogations qui subsistent.

La syntaxe des expressions indéfinies est de type général $X$ (de) $N$, avec une propriété assez générale, celle de la pronominalisation de la partie de $N$ sous la forme du clitique en, même lorsque le de n'est pas apparent :

\section{On en a acheté plusieurs, de livres / On a acheté plusieurs livres}

On peut y rattacher deux catégories de constructions sans introducteur $\mathrm{X}$, celle des articles en de et de la construction partitive définie, soit respectivement :

\section{On a acheté des livres / On a bu de ce vin}

La seconde se distingue de la première par ses restrictions d'emploi (elle n'est guère appropriée qu'à la droite du verbe, en construction directe) et par la possibilité de faire alterner le composant morphologique interne de type le avec les

${ }^{11}$ Il faut ajouter à mes travaux contrastifs ceux des quelques étudiants qui ont adopté mon analyse, notamment Shuaijun Mallet-Jiang dans sa thèse sur les complétives en chinois (2012), Liu Yue dans son mémoire de master sur les comparatives en chinois, ou encore Louis-Martin Onguene Essono dans sa thèse de doctorat d'État de Yaoundé (2000) et d'autres publications, qui sont les premières à donner une analyse précise des structures syntaxiques de l'ewondo (langue bantoue du Cameroun) : Onguene Essono, 2004, 2012. 
autres déterminants définis. Elle est plus étroitement apparentée à la construction partitive à introducteur, de type $\mathrm{X}$ de (le/ce/mon) $\mathrm{N}$, et l'on peut y voir une construction à tête vide relevant de cette sous-catégorie.

On a adopté le point de vue défendu par Kupferman (2004), de l'unicité des constructions nominales en de ayant un déterminant $\mathrm{X}$, toutes ces constructions ayant de façon cruciale par rapport à d'autres constructions de type $\mathrm{X}$ de $\mathrm{N}$ (ou, si l'on préfère, $\mathrm{X}$ de NP / X de DP) les propriétés suivantes, qui sont d'ailleurs liées : les critères de sélection actanciels sont ceux du groupe nominal, pas de $\mathrm{X}$ :

J'ai acheté un tablier de soubrette vs J'ai acheté une soubrette (J.-Cl. Milner, 1978, p. 41)

J'ai acheté un kilo de pain = J'ai acheté du pain

et, contrairement à ce qui se passe avec la construction voisine dite partie-tout ${ }^{12}$, qu'illustre les roues de la voiture, on peut supprimer $\mathrm{X}$ dans la construction postverbale directe :

On a mangé un peu de ce gâteau / On a mangé de ce gâteau

On a vendu les roues de la voiture / *On a vendu de la voiture ${ }^{13}$

L'analyse que l'on propose se sépare de celle de Kupferman sur la catégorisation du de: on en fait non pas une tête de quantifiant, comme lui, mais un constituant autonome que l'on nomme 'partitif indéfini', dont le rôle sémantique est d'introduire un groupe nominal (ou un adjectif), comme un élément fragmental, non ensembliste, dans sa dénomination. On soutient l'idée, déjà avancée par d'autres (C. Dobrovie-Sorin, C. Beyssade, 2004), que de et ses composés ne contiennent pas en soi l'idée de la quantification, pas plus qu'ils ne signalent une partition sur un ensemble, comme l'a montré entre autres G. Kleiber (2008) :

\section{Pierre a des ennuis}

ne renvoie pas à un ensemble d'où serait extraite une certaine quantité d'ennuis.

Comme il y a des configurations syntaxiques qui diffèrent, entre les emplois d'articles de des et de, les constructions à tête de quantifiants adverbiaux, les constructions à tête nominale, les partitifs au sens habituel (à interprétation en-

${ }^{12}$ La relation partie-tout se caractérise par deux propriétés : la partie a une identité distincte, et la relation entre partie est tout est une relation d'appartenance au sens large : les roues de la voiture signifie la voiture a des roues. Dans la relation partitive des déterminants, ces deux propriétés sont absentes, le terme initial étant une prédication sur la quantité, la proportion, ou un sous-ensemble défini du complément : beaucoup d'étudiants ne signifie pas *des étudiants ont beaucoup, comme ceux de mes étudiants ne signifient pas *mes étudiants ont ceux.

${ }^{13}$ Dans ce sens, une interprétation partitive traitant « voiture » comme massif n'est pas exclue. 
sembliste pour le complément du de), on propose une famille d'emplois de ce partitif indéfini, qui sont hiérarchisés, parfois plus proches des prépositions, et parfois de type article. Cette famille d'emplois inclut les de qui signalent entre leur tête et leur complément une relation de partie à tout dans laquelle la partie n'a pas d'identité propre, restant de la nature de celle du complément, mais elle couvre aussi les emplois sans interprétation ensembliste, où le groupe nominal est présenté comme un ou plusieurs exemplaires ou fragments d'entités nombrables ou massives. La tête de la relation partitive peut aussi être sémantiquement définie : ceux des étudiants qui ont choisi le latin; de même le de des disloquées :

\section{Celui-là, de salut, je l'ai réussi. (R. Queneau)}

L'analyse des constructions de type $\mathrm{X}$ de (le/les) $\mathrm{N}$ telle que l'on vient de la présenter, distincte des autres constructions en de est donc un préalable indispensable à l'étude de la détermination indéfinie, et elle ne se limite pas aux têtes quantifiantes, comme le suppose l'analyse de Kupferman. Milner (1978) envisage l'existence concomitante d'une tête "Qualité » parallèle des têtes quantitatives dans les déterminants, avant de la rejeter. Il est vrai qu'il s'intéresse plutôt aux constructions «affectives » comme cet idiot de Luc. Plus généralement, les constructions à déterminants définis ont aussi des compléments de $N$ dans les disloquées à tête pronominale : non seulement les démonstratifs, comme ci-dessus, mais aussi les possessifs, où les miens, d'étudiants, alterne avec mes étudiants et les interrogatifs, où laquelle de fille alterne avec quelle fille. Il n'y a guère que les articles définis qui y échappent, étant toujours liés directement aux noms. Cependant, pour M. Gross (1977 : 133-134), le $N$ et ce $N$ sont issus d'une source pronominale celui de $N$ (que P), le complément propositionnel reflétant le contenu prédicatif qui autorise la lecture définie de le ou ce. J'ai proposé de voir dans l'article défini le, comme le proposait $\mathrm{M}$. Gross partant de lui, la forme cliticisée d'un nominal de base, ce qui est d'ailleurs conforme à sa source historique, forme systématiquement adjectivée, où je vois en termes prédicatifs un argument dont le nom commun ou l'adjectif qui suit est le prédicat. Il n'y a pas de forme forte qui soit exactement la source de ce le article, mais l'hypothèse de Gross d'une relation à celui est plausible encore dans les usages actuels. Ainsi, on ne dira pas *lui, de chien pour le chien, mais celui-ci, de chien, ou celui du voisin, de chien, semblent représenter assez exactement les équivalents à tête pronominale des définis $c e$ chien et le chien du voisin.

Les constructions adjectivales du déterminant existent aussi, comme on le sait, pour les déterminants indéfinis, mais elles alternent généralement avec des équivalents pronoms, et la construction typique des partitifs indéfinis, avec $d e$, est donc la plus représentée.

On peut admettre que dans la formule générale $\mathrm{X}$ de (le/les) $\mathrm{N}$ qui représente la détermination des groupes nominaux, une bonne partie du contenu $\mathrm{X}$ est un 
prédicat sous-jacent d'un argument nominal plus basique. Cela se remarque avec la négation : pas beaucoup de $N$, pas cinq $N$, pas le même $N$, voient la négation porter sur ce contenu interne au déterminant, pas sur la relation entre groupe nominal et le verbe. Ces relations prédicatives ne sont jamais explicitées, dans les groupes nominaux, et elles obligent à poser que leur argument est une forme plus élémentaire de déterminant. C'est ce que l'on observe quand on rend explicite la prédication dans le déterminant :

\section{Il est venu beaucoup d'étudiants}

suppose quelque chose comme :

\section{Des étudiants sont beaucoup à être venus}

Et le déterminant de base est alors l'indéfini des. De même, plusieurs étudiants, c'est la relation prédicative des étudiants sont plusieurs (à faire telle chose) ; diverses personnes : des personnes qui sont «diverses » (au sens : de qualité, provenance, etc. différenciée) ; un peu d'eau : de l'eau qui est en petite quantité. Il est généralement possible de reconstruire en synchronie ces relations prédicatives sous-jacentes, parfois il faut recourir à la diachronie ; dans certaines personnes, on ne retrouve pas une relation qui serait des personnes qui sont certaines, et il faut imaginer une relation du type des personnes sont l'identité, l'existence est assurée/connue, avec le passage diachronique par un stade épithète de certaines personnes. Dans certains cas, le déterminant est importé d'une relation adverbiale, donc où la prédication adjectivale a pour argument le nom " quantité » : énormément de gens, c'est évidemment des gens qui sont en quantité énorme. Cette reconstruction esquissée permet de voir que les indéfinis ont leur source dans une relation prédicative dont le sujet est soit du type de : des personnes, des gens, de l'eau, soit au singulier, l'article un. Il s'agit de ce que la grammaire appelle des « articles », et ce qui les caractérise pour nous, c'est qu'ils ne sont pas des prédications de quantité sur l'objet nominal, mais le résultat de la saisie immédiate d'une entité aux contours imprécis, soit non nombrable (article dit partitif), soit nombrable, avec le choix secondaire du singulier et du pluriel. On posera qu'il n'y a pas de quantification dans l'article un (suivant en cela D. Leeman, 2004 ou S. De Vogüé, 2006) ; ainsi, dans j'ai eu un choc en apprenant la nouvelle, personne ne posera la question combien? un seul? Il n'y a pas non plus de quantification dans des de Paul écrit des romans, Luc a des cheveux tout blancs (dans le sens où tous ses cheveux sont blancs) ${ }^{14}$. Les structures diffèrent cependant : un

${ }^{14}$ Le sens est le même que dans Luc a les cheveux blancs. Pourtant, il y a un article indéfini ; son utilisation relève de la partitivité, non de la quantification. L'emploi de des au lieu de les présente cheveux comme un objet nouveau, nombrable, en quantité indéterminée, et comme un fragment de l'entité nommée « cheveux ». Par contre cette phrase peut être quantifiée, si des prend le sens de 
article est syntaxiquement du type des cardinaux, donc analysable comme un-de$N P$, structure mise en évidence par la reprise pronominale en du nom, alors que les autres articles sont basés sur le " de " 'partitif indéfini', dont la fonction est de dénoter une quantité (non nombrable) ou un nombre indéterminé d'une substance caractérisable par son nom. Ce de article est évidemment issu, comme l'est l'article un du numéral, de la préposition de notamment utilisée pour les relations partie-tout. Il se combine avec un le/les qui dénote par son nombre soit le massif, soit le nombrable. On n'y verra pas, contrairement à d'autres, un défini générique, mais un simple « classifieur $»^{15}$, un support à base nominale du syntagme déterminant, ayant des propriétés spécifiques : disparition devant un adjectif dans le cas du nombrable, absence dans les constructions à tête de quantifieur devant le $d e$, absence dans la portée proche de la négation, pas d'alternance avec les autres déterminants définis. Ce qui est illustré par, respectivement :

\section{J'ai mangé de délicieux gâteaux / J'ai mangé des délicieux gâteaux qu'on vient de m'offrir \\ Beaucoup d'étudiants travaillent / Beaucoup des étudiants travaillent \\ Personne n'achète de tableaux de ce peintre / Personne n'achète des tableaux de ce peintre \\ J'ai lu des articles de lui / J'ai lu de ses articles}

Les constructions de gauche sont également sémantiquement homogènes : aucune ne suppose une partition entre un sous-ensemble activé par la prédication, qui serait en relation avec un ensemble de référence, comme c'est le cas à droite, au moins pour une interprétation. Pour cela, et l'on admettra qu'il est impossible ici de développer pleinement cette analyse, on a adopté, comme Kupferman, l'hypothèse de l'unicité du de des constructions partitives et à quantifieurs, mais on distingue, dans le terme avec lequel il est en composition, l'article défini et le classifieur : dans les constructions de droite ci-dessus, c'est l'article défini qui figure.

Cela ne veut pas dire que tous les de ont des propriétés identiques dans ces constructions : on y développe l'hypothèse d'une famille de valeurs strictement hiérarchisées $^{16}$, parfois cumulables, toujours morphologiquement limitées à une seule position syntaxique, en quelque sorte par une généralisation de la « loi de cacophonie » des anciennes grammaires. Contrairement à Kupferman, on ne voit donc pas en ce de la tête d'un catégorie $\mathrm{Q}$, pour « quantificateur » mais une tête partitive au sens où il y a existence d'entités en masse ou nombre indéterminé, de la catégorie du nom qui suit. La quantification, lorsqu'elle apparaît dans le

quelques. Il n'y aurait pas non plus de quantification dans Il a un nez aquilin, par rapport à Il a le nez aquilin, mais la description d'un objet nombrable singulier, sans prédication de nombre explicite.

15 J'emprunte le terme et l'analyse à Rowlett (2007 : 64) et Guéron (2003). L'article défini est l'emploi marqué de ce classifieur, repéré comme élément connu/identifiable.

${ }^{16}$ Suivant en cela une idée de Carlier et Melis (2006). 
déterminant, devant cette tête partitive, est l'objet, comme on l'a signalé ci-dessus, d'une opération prédicative, absente de la construction partitive isolée.

Je me suis intéressé, dans l'analyse de cette prédication des indéfinis, à l'axe de la spécification, autrement dit, de la relation qui s'établit entre le syntagme introduit par un indéfini avec le prédicat dont il est argument. Dans les contextes factifs réels, il y a normalement spécification, ce qui explique que le syntagme indéfini, à référence vague, prend de ce fait une référence précise qui explique la reprise de ce syntagme par du défini :

Un homme est entré dans le café. Il a demandé une bière.

Lorsque le contexte n'est pas spécifiant, la spécification est inopérante et l'opération de référenciation reste inaboutie ${ }^{17}$ :

Rien n'indique qu'un homme soit entré dans le café. *Il a demandé une bière.

Le syntagme garde alors sa signification en isolation, celle d'un élément non distinguable d'autres de même dénomination : un homme = quelque homme que ce soit.

Les syntagmes à quantification gardent les mêmes propriétés, simplement la spécification s'applique au groupe nombrable ou à la quantité massive :

Cinq hommes sont entrés dans le café. Ils (= les cinq hommes) ont commandé des bières.

J'ai mis un peu d'argent de côté. Il (= ce peu d'argent) me suffira pour payer la réparation.

Certaines séries lexicales d'indéfinis sont réservées à des contextes sans spécification, ou à spécification imprécise, hors du réel : ce sont en français les formes de type qui que ce soit, un $X$ quelconque, n'importe quel $X$, ainsi que les négatifs. La partition entre contextes épisodiques réels et contextes à polarité négative est insuffisante à décrire les différents degrés de la spécification incertaine ou absente, et l'on a eu recours à l'analyse plus détaillée des contextes de spécification que propose Haspelmath (1997). Les résultats de cet examen des formes indéfinies selon les contextes m'ont conduit à modifier le classement de ces formes tel qu'opéré par Haspelmath, pour en proposer un autre, plus conforme aux usages du français actuel.

Bien que j'aie essayé d'être aussi exhaustif que possible dans cet ouvrage, je trouverais certainement, pour en revenir à la vision prospective du maître d'œuvre

${ }^{17}$ La reprise par $i l$ reste possible, parce que la spécification opère sans aboutir à une référence positivement assertée, dans Rien n'indique qu'un homme soit entré dans le café et qu'il ait demandé une bière. 
de ce numéro, des prolongations à mon examen. Je me contenterai d'en signaler deux.

Le premier tient à l'analyse que je propose d'un de constituant unique, mais à emplois différenciés, dans les syntagmes à articles indéfinis et partitifs, comme dans les constructions à quantification, ainsi que devant les adjectifs. Il faudrait voir dans quelle mesure cet emploi particulier de la préposition à valeur élative, qui en fait un élément autonome dans les articles, ou une préposition faiblement liée à son terme recteur, fait partie d'un continuum qui la relierait non seulement au de de la construction partie-tout (les roues de la voiture) mais aussi à d'autres de, la marque de possession, ou même l'introducteur d'infinitif.

Le second tient aux spécificités sémantiques des indéfinis. Il y a peut-être d'autres axes que la spécification, et que la quantification, à explorer. On se souvient que Milner dans son livre de 1978, qui est toujours une des bases de l'approche des indéfinis, s'était aussi intéressé à ce qu'il nomme le « système qualitatif ». On s'est rendu compte dans cette synthèse sur l'indéfinition que les aspects non quantitatifs étaient importants dans l'analyse des syntagmes indéfinis. Ainsi, la série de formes n'importe quilquoi, quel est souvent employée dans ce sens, non pas tant comme expression de l'indifférenciation des individus que comme expression évaluative (par exemple dans l'expression C'est n'importe quoi ! nettement dévalorisante). Les relations d'identité/altérité comptent aussi dans le repérage référentiel. Avant de réaliser ce travail, je pensais par exemple que le même était un déterminant défini. Pas du tout, ou plus exactement pas toujours, puisque ce déterminant peut introduire un nouvel objet, non repéré référentiellement auparavant, et en construction existentielle, propriété typique des indéfinis :

\section{Il y a la même voiture que la tienne stationnée dans la rue}

L'analyse de la prédication enchâssée dans le déterminant permet de comprendre ces particularités : la même est en réalité attribut d'une construction existentielle :

\section{Il y a une voiture qui est la même que la tienne dans la rue}

En somme la même dans cet exemple est un qualificatif sur une prédication indéfinie sous-jacente, introduisant bien un nouvel élément dans le discours distinct de l'objet support de la comparaison.

Cela n'est pas toujours le cas. Dans cet autre exemple, le déterminant est défini, et même pourrait être supprimé :

La même tempête qui, l'autre jour, secouait notre bateau, a défoncé la digue et emporté la route. (H. Hoppenot, cité dans Cl. Muller, 2019, p. 294)

= La tempête, la même que celle qui... 
Un autre cas est plus incertain : dans l'exemple qui suit, la construction décrit un objet unique, mais à référence qui reste indéterminée, parce que le syntagme nominal respecte une des conditions de l'indéfinition, la nouveauté référentielle :

Pour gagner de la place, les enfants dormiront si possible dans la même chambre

Le locuteur peut ignorer tout de ce qu'est la chambre en question, le prédicat dans le déterminant justifie le défini, mais on peut dire avec le même sens :

\section{Les enfants dormiront dans une même chambre}

La construction est donc " une chambre qui est la même », avec soit maintien de l'article un, soit substitution comme déterminant du prédicat le même à l'article. L'emploi de la même, dans cet énoncé, renvoie cette fois à un objet unique, et non similaire. C'est bien une condition de définitude, mais est-ce suffisant, puisque d'autres propriétés comme la présupposition d'existence ne sont pas assurées ?

La question est la même pour certains superlatifs, qu'il est parfois proposé d'analyser comme indéfinis :

\section{Il a le chien le plus intelligent du quartier}

Ici, de même, il n'y a pas de référence antérieure, le sens étant celui de la construction à relative :

\section{Il a un chien qui est le plus intelligent du quartier}

Il s'agit encore d'un déterminant prédicat sous-jacent, dont le le tient au superlatif, qui impose l'unicité, et qui vient se superposer à l'article $u n$ : un défini par intégration, sur une base indéfinie, pourrait-on dire. Une incertitude d'un type analogue apparaît dans un défini comme la majorité :

La majorité des Français soutient le président

La majorité des Français refuse le recul de l'âge de la retraite

Ces deux syntagmes nominaux où figure le déterminant partitif la majorité sont strictement identiques, mais on admettra qu'ils n'ont pas un contenu semblable : les Français qui constituent l'ensemble sur lequel opère le déterminant n'ont aucune raison d'être les mêmes, et en proportion identique. Cependant, dans leur contexte, chacun de ces syntagmes nominaux réalise bien un sous-ensemble unique, et défini par opposition au reste, qui ne peut être aussi une "majorité ». L'indéfinition est possible, une majorité pouvant ici être utilisé dans les deux 
cas, et comme précédemment, la relation est attributive : " une proportion des Français qui est la majorité ». Mais l'unicité ne coïncide pas avec une identité de proportions, elle décrit des situations qui varient à chaque occurrence. Le caractère défini d'un tel syntagme est donc tout à fait discutable, sauf à admettre que la définition tient strictement à l'unicité réalisée dans un contexte donné.

\section{Un mot pour conclure}

On voudra bien excuser ce qu'il y a d'un peu trop personnel dans les idées et les quelques illustrations de phénomènes linguistiques présentées ici. Comme je l'ai dit au début de ce texte, la thématique du numéro invitant à une vision prospective ne peut se fonder que sur un bilan, celui des recherches que chacun a eu l'occasion d'entreprendre, et en l'occurrence, il s'agit des miennes. Il me reste à souhaiter à mes successeurs dans ces domaines autant de plaisir que j'en ai eu à les parcourir, en évitant les défauts majeurs des jeunes chercheurs, l'absence de curiosité pour ce qui a pu être fait avant eux, et l'esprit étroit de certaines chapelles qui se contentent trop facilement des lectures de leur paroisse.

\section{Références citées}

Attal, P. (1979). Négation et quantificateurs. Thèse de doctorat d'État, Université de ParisVIII.

Attal, P. (1992). Commentaire critique de A Natural History of Negation, Laurence Horn, Chicago University Press, 1989. Langue française, 94, 103-122.

Attal, P. (1994). Questions de sémantique. Louvain, Peeters.

Carlier, A., \& Melis, L. (2006). L'article partitif et les expressions quantifiantes du type peu de contiennent-ils le même de ? In G. Kleiber, C. Schnedecker \& A. Theissen (Éds), La relation partie-tout (p. 449-464). Louvain, Peeters.

Chevalier, J. C. (2006). Combat pour la linguistique, de Martinet à Kristeva. Paris, ENS Éditions.

Creissels, D. (2006). Syntaxe générale, une introduction typologique (Vol. 2). Paris, Lavoisier.

Damourette, J., \& Pichon, E. (1911-1940). Des mots à la pensée. Essai de grammaire de la langue française. Paris, D'Artrey.

De Vogüé, S. (2006). L'article un, la position du sujet et la relation avec le prédicat. In F. Corblin, S. Ferrando \& L. Kupferman (Éds), Indéfinis et prédication (p. 265278). Paris, Presses de l'Université Paris-Sorbonne. 
Dobrovie-Sorin, C., \& Beyssade, C. (2004). Définir les indéfinis. Paris, CNRS Éditions.

Ducrot, O. (1984). Le dire et le dit. Paris, Éditions de Minuit.

Frege, G. (1971). Écrits logiques et philosophiques (Cl. Imbert, Trad.). Textes de 1879 à 1925, traduits de l'allemand, Paris, Seuil.

Gaatone, D. (1971). Étude descriptive du système de la négation en français contemporain. Genève, Droz.

Gerdes, K., \& Muller, Cl. (Éds). (2006). Ordre des mots et topologie de la phrase française. Linguisticae Investigationes, 29(1).

Givón, T. (1978). Negation in Language: Pragmatics, Function, Ontology. In P. Cole (Ed.), Syntax and Semantics. Pragmatics (Vol. 9, p. 69-112.). New York, Academic Press.

Gross, M. (1977). Grammaire transformationnelle du français. Syntaxe du nom. Paris, Larousse.

Guéron, J. (2003). Inalienable possession and the interpretation of determiners. In M. Coene \& Y. D'Hulst (Eds.), From NP to DP. The expression of possession in noun phrases (Vol. 2, p. 189-220). Amsterdam, John Benjamins.

Harris, Z. (1976). Notes du cours de syntaxe. Paris, Seuil.

Harris, Z. (1991). A Theory of Language and Information. Oxford, Clarendon Press.

Haspelmath, M. (1997). Indefinite Pronouns. Oxford, Oxford University Press.

Heldner, C. (1981). La portée de la négation. Stockholm, Norstedts.

Horn, L. R. (2001). A Natural History of Negation (2nd ed.). Stanford, CSLI Publications. Hualde, J. I., \& Ortiz de Urbina, J. (Eds.). (2003). A Grammar of Basque. Berlin — New York, De Gruyter Mouton.

Jackendoff, R. S. (1972). Semantic Interpretation in Generative Grammar. Cambridge (Massachusetts), MIT Press.

Kahrel, P., \& van den Berg, R. (Eds.). (1994). Typological Studies in Negation. Amsterdam, John Benjamins.

Katz, J. (1972). Semantic Theory. New York, Harper and Row.

Kleiber, G. (2008). Article partitif : une histoire vraiment ambiguë. In S. Reinheimer Ripeanu (Éd.), Studia Linguistica in Honorem Mariae Manoliu (p. 152-163). Bucarest, Editura Universitatii din Bucuresti.

Klima, E. (1964). Negation in English. In J. Fodor \& J. Katz (Eds.), The Structure of Language (p. 246-323). Englewoods Cliffs, Prentice Hall.

Kupferman, L. (2004). Le mot «de». Domaines prépositionnels et domaines quantificationnels. Bruxelles, De Boeck/Duculot.

Leeman, D. (2004). Les déterminants du nom en français, syntaxe et sémantique. Paris, Presses universitaires de France.

Li, C. N., \& Thomson, S. A. (1989). Mandarin Chinese, a Functional Reference Grammar. Berkeley, University of California Press.

Liu, Y. (2012). Constructions comparatives en chinois. Mémoire de master, Université de Bordeaux-3.

Mallet-Jiang, S. (2012). La complétive objet en chinois. Thèse, Université de Bordeaux-3. Martin, R. (1966). Le mot « rien » et ses concurrents en français. Paris, Klincksieck.

Martinet, A. (1985). Syntaxe générale. Paris, Armand Colin.

Milner, J.-Cl. (1978). De la syntaxe à l'interprétation. Paris, Seuil. 
Muller, Cl. (1975). Grammaire générative du français : la négation et les quantificateurs. Thèse, Université de Paris-III.

Muller, Cl. (1987). La négation en français, syntaxe, sémantique et interprétation avec les autres langues romanes. Thèse de doctorat d'État, Université de Paris-7.

Muller, Cl. (1991). La négation en français. Genève, Droz.

Muller, Cl. (1996). La subordination en français. Paris, Armand Colin.

Muller, Cl. (2008a). La négation, un opérateur transversal. De lingua latina, 1, 1-21.

Muller, Cl. (2008b). Les bases de la syntaxe ( $2^{\mathrm{e}}$ éd.). Pessac, Presses universitaires de Bordeaux.

Muller, Cl. (2017). La négation : le " côté obscur » de la référence, effets pragmatiques et conséquences grammaticales. In E. Hilgert et al. (Éds), Res per nomen V, Négation et référence (p. 121-137). Reims, Epure.

Muller, Cl. (2019). Indéfinis et partitifs en français. Pessac, Presses universitaires de Bordeaux.

Muller, Cl., et al. (Éds). (2001). Clitiques et cliticisation. Paris, Champion.

Nølke, H. (1992). « Ne pas », négation descriptive ou polémique ? Contraintes formelles pour son interprétation. Langue française, 94, 48-67.

Nourissier, Fr. (2000). À défaut de génie. Paris, Gallimard.

Onguene Essono, L. M. (2000). Subordonnées relatives et interrogatives en français et en ewondo. Thèse de doctorat d'État, Université de Yaoundé I, Cameroun.

Onguene Essono, L. M. (2004). Syntaxe et fonctionnement de la relative et de l'interrogative en ewondo. Essai d'analyse de la subordination en bantou. Revue internationale des arts, lettres et sciences sociales, 1(1), 113-139.

Onguene Essono, L. M. (2012). La phrase simple ewondo. Yaoundé, Éditions du Cerdotola.

Rebuschi, G. (1990). On the non-configurationality of Basque and some related phenomena. Anuario del Seminario de filologia vasca "Julio de Urquijo” (ASJU), 24(2), $351-383$.

Rowlett, P. (2007). The Syntax of French. Cambridge, Cambridge University Press.

Searle, J. R. (1972). Les actes de langage [traduction de Speech Acts, 1969]. Paris, Hermann.

Steinberg, D., \& Jakubovits, L. (Eds.). (1971). Semantics. Cambridge, Cambridge University Press.

Wittgenstein, L. (1961). Tractatus logico-philosophique, suivi de Investigations philosophiques (P. Klossowski, Trad.). Paris, Gallimard. 